\title{
A Survey of Requirements Management in Smart Product-Service Systems
}

\author{
Zuoxu WANG ${ }^{\mathrm{a}, 1}$, Pai ZHENG ${ }^{\mathrm{a}, \mathrm{b}}$, Chun-Hsien CHEN $^{\mathrm{a}}$ and Li Pheng KHOO ${ }^{\mathrm{a}}$

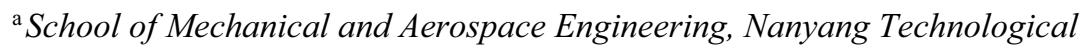 \\ University, Singapore 639798 \\ ${ }^{\mathrm{b}}$ Delta-NTU Corporate Laboratory for Cyber-Physical System, School of Electrical \\ and Electronic Engineering, Nanyang Technological University, Singapore 639798
}

\begin{abstract}
Driven by the advances of information and communication technologies (ICT) as well as the increasing complexity of product and service bundles in business market, smart product-service system (Smart PSS) has become a key factor for industrial companies to meet demanding customer expectations of individual product and services. Requirement management, as the first step of Smart PSS development, significantly affects its final success. In Smart PSS, requirement management is endowed with new capabilities and opportunities due to the mature of the advances of techniques and smart devices. The manner of requirement management has transformed from a human-intensive manner to a data-driven manner. Facing this challenge, scarcely any paper discussed the foundations of managing requirements in the context of Smart PSS. In this paper, the prerequisites of managing requirements in Smart PSS are discussed, data properties, representation format and reasoning methods are analyzed based on a data-driven requirement management architecture. Two potential scenarios of requirement management are discussed as well. This work depicts the deserving manner of requirement management in Smart PSS, which points out key points of managing requirements from heterogeneous usage data in the new Smart PSS paradigm.
\end{abstract}

Keywords. Product-service Systems, Requirement Management, Context-aware, Usage Stage

\section{Introduction}

Nowadays, the advances of information and communication technologies (ICT) and smart connected products (SCPs) have transferred the traditional product-service system (PSS) to a new PSS paradigm named smart product service system (Smart PSS) [1]. Many industrial companies, such as Rolls-Royces, Michelin and Xerox, have launched their smart services into market. In Smart PSS, large-volume and high varieties of data has capability to be collected and processed by mobile crowd sensing (MSC) techniques [2] and big data techniques[3][4]. Ubiquitous connectivity can be achieved by Internet of Things (IoT) [5]. At the same time, with the support of cloud computing platforms [6] and embedded system integrating hardware, sensors, microprocessors and software into SCPs, online-offline smartness is capable of implementation as well [7]. These

\footnotetext{
${ }^{1}$ Corresponding author, Email: zuoxu001@e.ntu.edu.sg.
} 
technological advances make requirement management in Smart PSS as a data-driven manner rather than human-intensive manner which relies on small-scale customer survey data [8]. Meanwhile, from market-driven perspective, since users are involved into the total process of value delivery by generating user-generated contents (UGC) and product-sensing data, the requirement management process becomes a value-co-creation manner. Facing the significant challenges of requirement management in Smart PSS, scarcely any report discussed how to model requirements in the context of Smart PSS. To fulfill this gap, this paper aims to study the foundations and prerequisites of requirement management in Smart PSS.

The rest of this paper is organized as follows. Related works about requirement management in PSS are reviewed in Section 1. Section 2 introduced the prerequisites of requirements management in Smart PSS. A data-driven requirement management architecture is discussed from the information flow perspective in Section 3, followed by two potential scenarios of requirement management in Smart PSS in Section 4. Finally, Section 5 summaries the conclusions, limitations and future works of this paper.

\section{Related works}

Smart PSS is a digital-based ecosystem of stakeholders, tangible smart devices and intangible services for value co-creation with characteristics of high complexity, dynamics and interconnectedness [9]. In the early studies of Smart PSS, Valencia et al. [10] addressed several distinctive elements in Smart PSS development by analyzing cases and interviewing PSS-related practitioners. The elements of Smart PSS, including complex marketing offering, closer interactions between designers and end-users, high dependency of context and the ever-evolving of the Smart PSS value proposition were discussed in their work. Furthermore, the specific influences of technical advances in Smart PSS were studied as well. For example, the product lifecycle including production design, manufacturing, after-sale services and so on which is enabled by digital twin is discussed by Tao et al. [11]. Smarter devices, e.g. smart, connected open architecture product (SCOAP) [12] is also able to be realized through cloud computing platforms, virtual twin, IoT and big data processing techniques. Meanwhile the advanced services, such as remote monitoring [13], remote maintenance [4] and smart logistic and packaging [6] are all addressed to have potential opportunities of creating business profits for companies.

Although Smart PSS enabled many advanced services, the existing research of Smart PSS mainly focuses on the new product-service generation or service innovation beginning from the early design stage in a forward-manner instead of improving or reconfiguring product-service design in an inverse backward-manner. To the author's knowledge, only Hou and Jiao [8] presented a closed-loop decision framework for the inverse design based on product usage data in detail. Hence, the approaches of Smart PSS design improvements during usage stage still remains to be explored.

Furthermore, requirement management in conventional PSS design basically contains four parts, namely requirement elicitation, requirement analysis, requirement specification and requirement forecast [14]. However, elicitation of requirements is usually conducted through focus group, structured/semi-structured interview, questionnaire and user experience test, etc., which only extracting extrinsic user behaviors and expectation but omitting the implicit and intrinsic customer requirements [2][8]. Meanwhile, the analysis and specification of requirements are almost based on 
small-scale user survey data in a human-intensive manner. In Smart PSS, large-volume user-generated and product-sensing data can be collected. User behavior patterns and user mind expectations are all embraced among those objective data, making the requirement management as a data-driven manner. Nevertheless, there is still lack of requirement management research based on usage data in Smart PSS.

\section{Prerequisites of requirements management in Smart PSS}

In this section, some prerequisites of requirement management will be discussed as the foundation for further study. Figure 1 displays the comparison of conventional PSS business model and Smart PSS business model. Some differences of PSS and Smart PSS in terms of PSS development can be derived as follows.

1. Smart PSS becomes a closed-loop system. After-sale services and capabilities, including smart use, smart reuse, smart remanufacturing and smart recycling, are enabled by smart enablers [15]. By combining those after-sale services and the relevant product-lifecycle data into the consideration of Smart PSS design, the circularity of the ecosystem provides cost reduction and material reduction and new potential business revenues.

2. At the same time, pain points of Smart PSS appearing in the usage stages are also able to be discovered from user -generated contents or product-sensing data and to be solved by reconfiguring the smart devices or services or offering new services, making Smart PSS as an ever-evolving system. In this way, the dynamic requirements are required to be managed as well.

3. Context-awareness should be considered in Smart PSS. Valencia et al. [10] has underlined the high dependency of context-awareness of Smart PSS. Since Smart PSS aims to provide customized value propositions to fulfill individual requirements, the specific context of the scenarios, usage environment and the performance of products and services should be under control and real-time monitored. Only in this way, suitable product and service bundle can be customized and delivered to individuals in time.

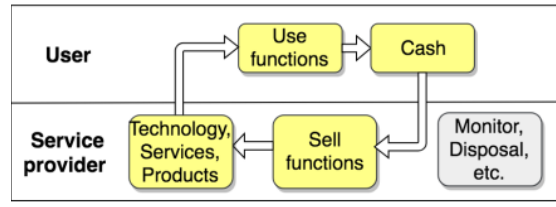

PSS business model

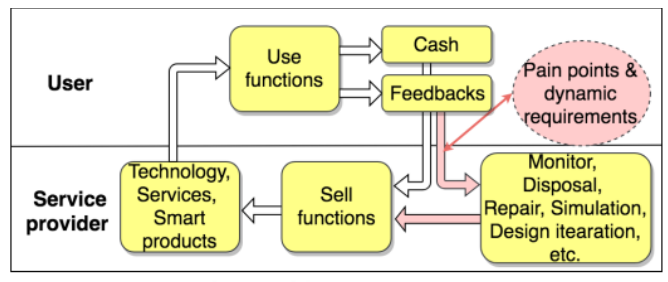

Smart PSS business model

Figure 1. A comparison between conventional PSS and Smart PSS.

Base on the distinctions of Smart PSS, we can see that the usage stage and end-oflife stage have dramatical changes. Requirement management also extend its influence from just early design stage to usage stage. Three prerequisite of requirement modeling are addressed here.

1. Usage data should be collected and processed.

2. Heterogeneous data and information.

3. Highly context dependency. 
As a summary, the requirement management in Smart PSS is changed from data volume, data types, data resources to the implications of extracted information and knowledge. The methods of organizing and processing those data also might be adjusted correspondingly.

\section{A data-driven requirement management architecture}

In this section, a 4-layer data-driven requirement management architecture is presented and analyzed from the information flow perspective, as shown in Figure 2. How to collect and organize heterogeneous data is discussed in data level and representation level. Requirements are extracted and analyzed in reasoning level, transforming data to meaningful information. In addition, requirement prioritization and specification also sit in reasoning level to identify the importance and correlations of requirements. At last, similarity and quality of extracted requirement need to be evaluated for requirement query and reuse so it is located in evaluation level. Knowledge among the certain Smart PSS has opportunities to be determined if the extracted requirements as information are validated in the same or similar scenarios/context. More details will be discussed layer by layer via analyzing the existing relevant works.

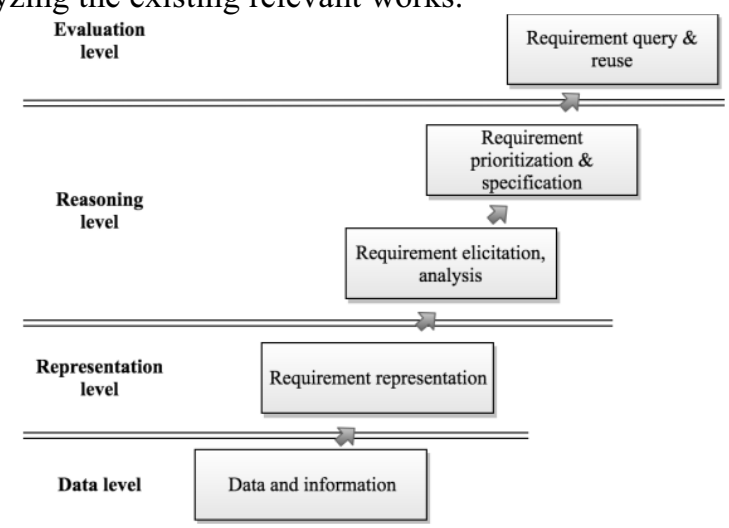

Figure 2. Requirement management architecture from the information flow perspective.

\subsection{Data and information applied for requirement management in Smart PSS}

The studies about data and information applied for requirement management are compared from three perspectives, namely data volume, data resource and data format, as shown in Table 1. As for data volume, Dey and Lee [16] proposed a methodology called REASSURE which is based on repertory grids to acquire knowledge from experts and users. This method can only suit for small-scale problems because the scores are given by human, making it human-intensive. However, the trend of scaling data volume in requirement management has been found by some researchers, for example Huang et al. [17] and Wang et al. [18]. Furthermore, the data resources are changed from stakeholders (e.g. domain experts, customers) to more comprehensive system-in-use data. Meanwhile, the data format also transformed from scores or ratings to various formats including natural language, vectors and graphs. As a summary, requirement management is facing radical transforms since the basic features of data and information have already been changed in Smart PSS. 
Table 1. The comparison of data properties between conventional requirement management (RM) and the one in Smart PSS.

\begin{tabular}{lllll}
\hline & Reference & Data volume & Data resource & Data format \\
\hline Conventional RM & {$[16]$} & Small-scale & $\begin{array}{l}\text { Domain experts, } \\
\text { general users }\end{array}$ & Numerical (scores) \\
& {$[19]$} & $/$ & Stakeholders & Numerical (scores) \\
& {$[20]$} & $/$ & Customers & Numerical (scores) \\
& {$[17]$} & Large-scale & User request & Natural language \\
RM in Smart PSS & {$[21]$} & $/$ & System-in-use data & Numerical (matrix) \\
& {$[18]$} & Large-scale & System-in-use data & Numerical (graph with vectors) \\
\hline
\end{tabular}

\subsection{Representation formats of requirements in Smart PSS}

Standardizing data and information into a uniform representation format is the prerequisite of handling requirements, especially for Smart PSS which is involved in heterogeneous data. To the authors' knowledge, the representation formats can be generally divided into four groups, i.e. hierarchical structure, natural language, vector, matrix or tensors and graph-based representation. Each of representation format has their own merits and defects, making them suitable to coping with different problems, as shown in Table 2.

Table 2. A summary of requirement representation format in PSS.

\begin{tabular}{|c|c|c|c|}
\hline $\begin{array}{l}\text { Representation } \\
\text { format }\end{array}$ & Reference & Merits & Defects \\
\hline Hierarchical structure & {$[22][23][24][25]$} & $\begin{array}{l}\text { A systematic way to link } \\
\text { requirements from abstract } \\
\text { level to detailed level }\end{array}$ & $\begin{array}{l}\text { Problematic to conduct } \\
\text { large-scale computational } \\
\text { analysis }\end{array}$ \\
\hline Natural language & {$[26][27]$} & $\begin{array}{l}\text { Intuitive for human- } \\
\text { understanding }\end{array}$ & $\begin{array}{l}\text { Need capacity for } \\
\text { machine-readability }\end{array}$ \\
\hline $\begin{array}{l}\text { Vector, matrix or } \\
\text { tensors }\end{array}$ & $\begin{array}{l}{[28][29][30]} \\
{[31]}\end{array}$ & Can be handled in large-scale & $\begin{array}{l}\text { Might be high } \\
\text { dimensional; might not be } \\
\text { suitable to all types of data }\end{array}$ \\
\hline Graph & [32] [33] [34] & An intuitive and general way & / \\
\hline
\end{tabular}

Hierarchical structure is the conventional format to manage requirements in both software engineering and product design and development. Many studies have chosen it as the representation way. It has the advantage that it is a systematic methodology to divide abstract requirements into several detailed and hierarchical requirements, making it easy and organized to manage requirements for complex systems. However, it is problematic to conduct large-scale computational analysis.

Natural language as the intuitive way for human to express their requirements, nowadays also has capability of extracting valuable information and knowledge for requirement management with the assistance of natural language processing (NLP) techniques. Nevertheless, this format is not suitable for RE in Smart PSS because NLP techniques can only handle single format, i.e. natural language, which does not fulfill the prerequisite of processing heterogeneous data in Smart PSS. 
Vector, matrix or tensors can be applied to handle large-scale requirements in complex systems, although its corresponding reasoning methods need to deal with high dimensions.

Graph is an intuitive way to represent complex system. It can contain various information due to its simple representation way. Hence it has potential to represent information which comes from heterogeneous data resource with various data formats.

As a result, graph is a feasible way to represent requirements in Smart PSS. Hybrid methods which combine graph representation and other formats are also worth further studies.

\subsection{Reasoning methods of requirement elicitation, prioritization and specification}

Reasoning layer basically contains requirement elicitation, prioritization and specification. Analytic hierarchical process (AHP)/analytic network process (ANP)based, fuzzy theory/rough set-based, NLP-based and deep learning-based are four typical types of reasoning methods appeared in relevant literatures, as shown in Table 3.

Table 3. A summary of reasoning methods of requirement elicitation, prioritization and specification.

\begin{tabular}{|c|c|c|c|}
\hline Reasoning method & Reference & Merits & Defects \\
\hline AHP/ANP-based & {$[19][20]$} & $\begin{array}{l}\text { Simple computational } \\
\text { process }\end{array}$ & $\begin{array}{l}\text { Omit the correlationship } \\
\text { between elements }\end{array}$ \\
\hline $\begin{array}{l}\text { Fuzzy theory/rough set- } \\
\text { based }\end{array}$ & {$[19][37][38]$} & $\begin{array}{l}\text { Can deal with } \\
\text { uncertainty }\end{array}$ & $\begin{array}{l}\text { Need engineer to identify } \\
\text { the membership functions }\end{array}$ \\
\hline NLP-based & {$[17][43]$} & $\begin{array}{l}\text { Good performance in } \\
\text { specific tasks }\end{array}$ & $\begin{array}{l}\text { Cannot deal with various } \\
\text { types of data }\end{array}$ \\
\hline Deep-learning based & {$[39][40][41][42]$} & $\begin{array}{l}\text { Can deal with large- } \\
\text { scale problem }\end{array}$ & Need to train the model \\
\hline
\end{tabular}

AHP/ANP are conventional reasoning methods in requirement analytics. In PSS literature, many examples focus on the integration of QFD and AHP/ANP [20] [35][36]. Though these studies can be regarded as a forward step of requirement management in PSS field, the interactions between PSS components are still omitted.

Fuzzy theory/rough set-based methods have the distinctive merit of handling uncertainty. Since requirements are uncertain in nature, requirements can be different even if under similar scenarios [37][38], for example, low manufacturing tolerance is required while producing an airplane engine but the tolerance can be relatively broaden while producing paper cup. Even in the same scenario, the requirement will still need to be updated because of the change of environment property. Thus, fuzzy theory/rough set-based methods have significant roles in requirement management in PSS filed. It has potential to collaborate with other reasoning methods.

NLP-based methods have good performance in specific tasks, such as questionanswering task, sentiment analysis and so on. This technique is also utilized in requirement management. For instance, Huang et al. [17] utilized NLP to clarify crowdsourcing user requests by classify them into groups according to project-specific or non-project-specific keywords. Besides its good performance, it cannot deal with various types of data simultaneously, making it less useful in the context of Smart PSS.

Finally, deep learning-based methods are increasingly prosperous in numerous fields. Different deep learning algorithms are applied to solve various problems. For 
example, genetic algorithm has been utilized for customer requirement specification [39]. Ant colony optimization is able to capture and select requirements [40][41]. And Artificial immune system (AIS) is capable of organizing requirements while the market environment changing [42].

Facing to the challenges of requirement management in Smart PSS aforementioned, fuzzy theory-based methods and deep learning-based methods have more strengths for the reasoning task in Smart PSS.

\subsection{Requirement reuse in Smart PSS}

The requirements extracted and analyzed from heterogeneous data are only information, only those requirements which are examined by experts or practical cases can become knowledge. Though the topic of knowledge reuse has been widely discussed in manufacturing system and product development, only few reports study how to reuse requirement in Smart PSS. In order to implement requirement reuse, the similarity of PSS cases are examined based on the context-based activity model in Wu et al's study[44]. Akmal et al.[45] proposed an ontology-based approach that can determine the similarity between two classes using feature-based similarity measures that replace features with attributes. More detailed studies are still required for the requirement reuse in Smart PSS.

\section{Potential scenarios}

In order to illustrative the to-be scenarios of requirement management in Smart PSS, two examples in different fields, i.e. smart manufacturing and smart living are addressed as follows.

\subsection{Smart design}

As shown in Figure 3 (a), heterogeneous data including saddle pressure and cycling distance as product-in-use data and environment humidity as context information can be collected through embedded sensors on smart bikes during usage stage. All the information can be utilized to find out the relevant factors which can improve riding performance under different scenarios as requirements. For example, by analyzing the correlation between environmental humidity and saddle performance, the implicit requirement that redesigning the padding of saddle is required at rainy area. Then relevant services, e.g. reconfiguring the padding material as super dry material, can be provided to users living in rainy area.

\subsection{Smart living}

The to-be requirement management scenario is also suitable for smart living. Supposed a service provider offer a service that recommending best restaurant based on not only user ratings, but also weather, location, numbers of customers, expected waiting time and so on, as shown in Figure 3 (b), the advanced service become personalized based on the user's own preference and their current location and weather instead of just based 
on historical orders and others' comments. Context-awareness and real-time requirement acquisition can be achieved in this smart living scenarios.

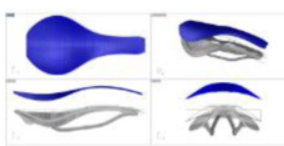

A generated service: Reconfigure the design of bike saddle

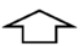

Requirement: identify the influential factor for riding performance

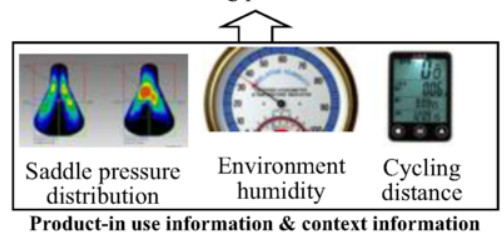

(a) Smart manufacturing

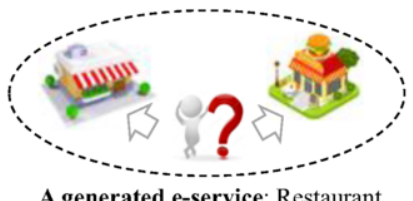

A generated e-service: Restaurant recommendation based on context info and service info

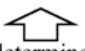

Requirement: determine a suitable (e.g. considering weather, distance and taste) restaurant for users

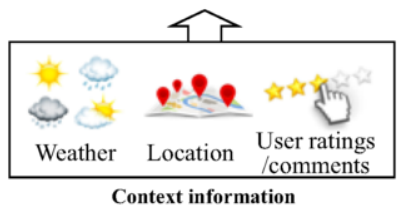

(b) Smart living

Figure 3. Two potential to-be scenarios of requirement management in Smart PSS.

Hence, by considering heterogeneous usage data including context information into requirement management, requirements can be extracted and managed with more accuracy and lower stakeholders' subjectivity, consequently better services which fulfill individual requirements can be offered to users as well.

\section{Conclusion}

With the advances of technologies and smart devices, advanced services are offered to users for the aim of satisfying their induvial requirements, which comprise the Smart PSS. Higher complexity, closer interactions between service providers and end-users and ever-evolving value proposition are able to be implemented by those smart enablers (e.g. virtual twin, IoT, cloud computing and big data technique) in Smart PSS. In this context, requirement management transfer its conventional human-intensive manner to a datadriven manner. This paper focuses on the basis of how to manage and organize requirements from heterogeneous usage data. The contributions of this paper lie in two aspects:

1. Three prerequisites of modeling requirements in Smart PSS were introduced. By introducing the prerequisites of requirement management, those basic properties such as data resources and data types can be generally determined, Then the reasoning method for subsequent analysis and specification can be identified as well.

2. A 4-layer data-driven requirement management architecture was proposed. Some research findings are derived by comparing the existing studies in each requirement management task. These findings describe a deserving manner of requirement management in Smart PSS.

Moreover, two potential to-be scenarios of requirement management are presented as well. Besides these contributions, some limitations still lie in this paper. More practical 
cases remain to validate the supposed requirement manner. And an in-depth systematic requirement management framework is still required further discussion. This paper is hoped to be regarded as the foundation of requirement management in Smart PSS.

\section{Acknowledgement}

This work was conducted within the Delta-NTU Corporate Lab for Cyber-Physical Systems with funding support from Delta Electronics Inc. and the National Research Foundation (NRF) Singapore under the Corp Lab@University Scheme.

\section{Reference}

[1] M. Zheng, X. Ming, L. Wang, D. Yin, and X. Zhang, Status Review and Future Perspectives on the Framework of Smart Product Service Ecosystem, Procedia CIRP, vol. 64, pp. 181-186, 2017.

[2] P. Zheng, X. Xu, and C.-H. Chen, A data-driven cyber-physical approach for personalised smart, connected product co-development in a cloud-based environment, J. of Int. Manuf., pp. 1-16, 2018.

[3] F. Tao and Q. Qi, New IT Driven Service-Oriented Smart Manufacturing: Framework and Characteristics, IEEE Transactions on Systems, Man, and Cybernetics: Systems, vol. 49, no. 1, pp. 81-91, Jan. 2019.

[4] J. Lee, H.-A. Kao, and S. Yang, Service Innovation and Smart Analytics for Industry 4.0 and Big Data Environment, Procedia CIRP, vol. 16, pp. 3-8, 2014.

[5] T. Takenaka, Y. Yamamoto, K. Fukuda, A. Kimura, and K. Ueda, Enhancing products and services using smart appliance networks, CIRP Annals, vol. 65, no. 1, pp. 397-400, 2016.

[6] Y. Zhang, S. Liu, Y. Liu, and R. Li, Smart box-enabled product-service system for cloud logistics, International Journal of Production Research, vol. 54, no. 22, pp. 6693-6706, Nov. 2016.

[7] P. Zheng, T.-J. Lin, C.-H. Chen, and X. Xu, A systematic design approach for service innovation of smart product-service systems, J. Clean. Prod., vol. 201, pp. 657-667, 2018.

[8] L. Hou and R. J. Jiao, Data-informed inverse design by product usage information: a review, framework and outlook, Journal of Intelligent Manufacturing, pp. 1-24, 2019.

[9] B. Kuhlenkötter et al., New Perspectives for Generating Smart PSS Solutions - Life Cycle, Methodologies and Transformation, Procedia CIRP, vol. 64, pp. 217-222, 2017.

[10] A. Valencia Cardona, R. Mugge, J. Schoormans and H. Schifferstein, Challenges in the design of smart product-service systems (PSSs): Experiences from practitioners, Proc. of the 19th DMI: Academic Design Management Conference. Design Management in an Era of Disruption, London, Sept 2-4, 2014.

[11] F. Tao, J. Cheng, Q. Qi, M. Zhang, H. Zhang, and F. Sui, Digital twin-driven product design, manufacturing and service with big data, The International Journal of Advanced Manufacturing Technology, vol. 94, no. 9-12, pp. 3563-3576, Feb. 2018.

[12] P. Zheng, Y. Lin, C.-H. Chen, and X. Xu, Smart, connected open architecture product: an IT-driven cocreation paradigm with lifecycle personalization concerns, Int. J. Prod. Res., 57:8, pp. 2571-2584, 2019.

[13] T. Grubic and I. Jennions, Remote monitoring technology and servitised strategies - factors characterising the organisational application, Int. J. of Prod. Res., vol. 56, no. 6, pp. 2133-2149, 2018.

[14] W. Song, Requirement management for product-service systems: Status review and future trends, Computers in Industry, vol. 85, pp. 11-22, 2017.

[15] A. Alcayaga, M. Wiener, and E. G. Hansen, Towards a framework of smart-circular systems: An integrative literature review, Journal of Cleaner Production, vol. 221, pp. 622-634, Jun. 2019.

[16] S. Dey and S.-W. Lee, REASSURE: Requirements elicitation for adaptive socio-technical systems using repertory grid, Information and Software Technology, vol. 87, pp. 160-179, 2017.

[17] C. Li, L. Huang, J. Ge, B. Luo, and V. Ng, Automatically classifying user requests in crowdsourcing requirements engineering, Journal of Systems and Software, vol. 138, pp. 108-123, Apr. 2018.

[18] Z. Wang, P. Zheng, C. Chen, and L. Khoo, A graph-based requirement elicitation approach in the context of smart product-service systems, 48th International Conference on Computers and Industrial Engineering, Auckland, New Zealand, 2018.

[19] T. T. Sousa-Zomer and P. A. C. Miguel, A QFD-based approach to support sustainable product-service systems conceptual design, The International Journal of Advanced Manufacturing Technology, vol. 88, no. 1-4, pp. 701-717, 2017. 
[20] M. Fargnoli and N. Haber, A practical ANP-QFD methodology for dealing with requirements' inner dependency in PSS development, Computers \& Industrial Engineering, vol. 127, pp. 536-548, Jan. 2019.

[21] Z. Liu and X. Ming, A framework with revised rough-DEMATEL to capture and evaluate requirements for smart industrial product-service system of systems, International Journal of Production Research, pp. 1-19, Feb. 2019.

[22] T. Gorschek and C. Wohlin, Requirements abstraction model, Requirements Engineering, vol. 11, no. 1, pp. 79-101, 2006.

[23] E. Geisberger, M. Broy, J. Kazmeier, D. Paulish, and A. Rudorfer, Requirements engineering reference model (REM), Trchnical Report, TU Munich, 2006.

[24] K. Pohl and E. Sikora, COSMOD-RE: Supporting the co-design of requirements and architectural artifacts, 15th IEEE Int. Requirements Engineering Conference, 2007. RE'07., 2007, pp. 258-261.

[25] M. Berkovich, J.M. Leimeister, A. Hoffmann, and H. Krcmar, A requirements data model for product service systems, Requirements Engineering, vol. 19, no. 2, pp. 161-186, 2014.

[26] H. Eyal Salman, M. Hammad, A.-D. Seriai, and A. Al-Sbou, Semantic Clustering of Functional Requirements Using Agglomerative Hierarchical Clustering, Information, vol. 9, no. 9, p. 222, 2018.

[27] P. Laurent and J. Cleland-Huang, Lessons learned from open source projects for facilitating online requirements processes, International Working Conference on Requirements Engineering: Foundation for Software Quality, 2009, pp. 240-255.

[28] P. W. Murray, B. Agard, and M. A. Barajas, Forecast of individual customer's demand from a large and noisy dataset, Computers \& Industrial Engineering, vol. 118, pp. 33-43, 2018.

[29] C. I. Papanagnou and O. Matthews-Amune, Coping with demand volatility in retail pharmacies with the aid of big data exploration, Computers \& Operations Research, vol. 98, pp. 343-354, 2018.

[30] Y. Wang, S. Yu, and T. Xu, A user requirement driven framework for collaborative design knowledge management, Advanced Engineering Informatics, vol. 33, pp. 16-28, Aug. 2017.

[31] N. Misaghian and H. Motameni, An approach for requirements prioritization based on tensor decomposition, Requirements Engineering, vol. 23, no. 2, pp. 169-188, 2018.

[32] C.-H. Chen and L. G. Occeña, Knowledge decomposition for a product design blackboard expert system, Artificial Intelligence in Engineering, vol. 14, no. 1, pp. 71-82, 2000.

[33] Y. Kim and K. Suzuki, Social context representation in product-service systems with internet of things, Open Journal of Social Sciences, vol. 3, no. 07, p. 187, 2015.

[34] Y. Kim, E. Wang, S. Lee, and Y. Cho, A product-service system representation and its application in a concept design scenario, Proc. of the 1st CIRP Industrial Product-Service Systems (IPS2) Conf., 2009.

[35] X. Geng, X. Chu, D. Xue, and Z. Zhang, An integrated approach for rating engineering characteristics' final importance in product-service system development, Computers \& Industrial Engineering, vol. 59, no. 4, pp. 585-594, 2010.

[36] X. Geng, X. Chu, D. Xue, and Z. Zhang, A systematic decision-making approach for the optimal productservice system planning, Expert Systems with Applications, vol. 38, no. 9, pp. 11849-11858, 2011.

[37] A. K. Tripathy and P. K. Tripathy, Fuzzy QoS requirement-aware dynamic service discovery and adaptation, Applied Soft Computing, vol. 68, pp. 136-146, 2018.

[38] P. Zheng, X. Xu, S. Q. Xie, A weighted interval rough number-based method to determining relative importance ratings of customer requirements in QFD product planning, Journal of Intelligent Manufacturing, vol. 30, no. 1, pp. 3-16, 2019.

[39] R. Dou and C. Zong, Application of Interactive Genetic Algorithm based on hesitancy degree in product configuration for customer requirement, International Journal of Computational Intelligence Systems, vol. 7, no. sup2, pp. 74-84, 2014.

[40] J. del Sagrado, I. M. del Aguila, and F. J. Orellana, "Multi-objective ant colony optimization for requirements selection," Empirical Software Engineering, vol. 20, no. 3, pp. 577-610, 2015.

[41] Q. Yang, H. Jiao, F. Song, G. Pan, and D. Wei, Customer requirement acquisition system and requirement expression guidance based on ant colony optimization, Adv in Mech Eng, 2017, 9:6, 1687814017704412.

[42] Y. T. Chong and C.-H. Chen, Management and forecast of dynamic customer needs: An artificial immune and neural system approach, Advanced engineering informatics, vol. 24, no. 1, pp. 96-106, 2010.

[43] X. Deng and Y. Zeng, A Novel Framework for Product/Service Systems Using Environment-Based Design Methodology, ASME 2014 International Design Engineering Technical Conferences and Computers and Information in Engineering Conference, 2014, p. V004T06A025-V004T06A025.

[44] Y. Wu, J.-H. Lee, Y. S. Kim, S. W. Lee, S.-J. Kim and X. Yuan, A similarity measurement framework of product-service system design cases based on context-based activity model, Computers \& Industrial Engineering, vol. 104, pp. 68-79, 2017.

[45] S. Akmal, L.-H. Shih, and R. Batres, Ontology-based similarity for product information retrieval, Computers in Industry, vol. 65, no. 1, pp. 91-107, 2014. 